\title{
Modeling the skin pattern of fishes
}

\author{
Rafael A. Barrio, ${ }^{1,2}$ Ruth E. Baker, ${ }^{1, *}$ Benjamin Vaughan, Jr., ${ }^{1}$ Karla Tribuzy, ${ }^{3}$ Marcelo R. de Carvalho, ${ }^{4}$ \\ Rodney Bassanezi, ${ }^{5}$ and Philip K. Maini ${ }^{1,6}$ \\ ${ }^{1}$ Centre for Mathematical Biology, Mathematical Institute, University of Oxford, 24-29 St. Giles', Oxford OX1 3LB, United Kingdom \\ ${ }^{2}$ Instituto de Fisica, Universidad Nacional Autónoma de México (UNAM), Apartado Postal 20-364 01000 México, D.F., Mexico \\ ${ }^{3}$ Universidade Federal do Amazonas-UFAM/ICE, Av. Gen. Rodrigo Otavio Jordão Ramos 3000, CEP.: 69077-000 Manaus, AM, Brasil \\ ${ }^{4}$ Universidade de São Paulo, USP-Departamento de Zoologia, Instituto de Biociências, Rua do Matão, Trav. 14, no. 101, \\ São Paulo, CEP 05508-900, Brasil \\ ${ }^{5}$ Universidade Estadual de Campinas-UNICAMP/IMECC, Cidade Universitária Zeferino Vaz, Barão Geraldo, \\ CEP.: 13083-970 Campinas, SP, Brasil \\ ${ }^{6}$ Oxford Centre for Integrative Systems Biology, Department of Biochemistry, South Parks Road, Oxford OX1 3QU, United Kingdom
}

(Received 1 September 2008; revised manuscript received 11 February 2009; published 18 March 2009)

\begin{abstract}
Complicated patterns showing various spatial scales have been obtained in the past by coupling Turing systems in such a way that the scales of the independent systems resonate. This produces superimposed patterns with different length scales. Here we propose a model consisting of two identical reaction-diffusion systems coupled together in such a way that one of them produces a simple Turing pattern of spots or stripes, and the other traveling wave fronts that eventually become stationary. The basic idea is to assume that one of the systems becomes fixed after some time and serves as a source of morphogens for the other system. This mechanism produces patterns very similar to the pigmentation patterns observed in different species of stingrays and other fishes. The biological mechanisms that support the realization of this model are discussed.
\end{abstract}

DOI: 10.1103/PhysRevE.79.031908

PACS number(s): 87.18.Hf, 82.40.Ck, 82.40.Bj, 47.54.- $-\mathrm{r}$

\section{INTRODUCTION}

In 1952 Alan Turing proposed that a system of chemical substances, or morphogens, diffusing and reacting with each other, could produce spatially stable patterns [1]. He introduced a system of reaction-diffusion equations and determined general conditions for the formation of spatial patterns driven by diffusion. For many years the existence of the Turing mechanism for morphogenesis in real biological systems has been difficult to corroborate, although it seems that there is now strong experimental evidence that pattern of follicles on the skin of mice is produced by a Turing mechanism [2]. Kondo and Asai [3] suggested that the pigment patterns on the skin of the angelfish Pomacanthus imperator could be modeled by a Turing system, a notion further developed by us $[4,5]$. A further example of Turing systems in pattern formation can be found in the generation of left-right asymmetry in the mouse embryo [6].

Another major issue has been that Turing models give very simple patterns of spots or stripes, and more elaborate models are needed to mimic the patterns observed in many biological systems. Coupled systems of reaction-diffusion equations have successfully been applied in the modeling of a number of complex biological patterning events. From the arrangement of omatidia in the Drosophila retina [7] to the patterns on sea shells [8] and the arrangement of tentacles around the primary organizer in hydra [9]. It is this type of model that we discuss here.

\section{A. Linearly coupled Turing models}

A few years ago we proposed [10] that coupling two simple Turing systems could produce more complex patterns

\footnotetext{
*Corresponding author.
}

and simulate reasonably well some complicated structures, including the skin patterns of some fish species. There we used linear, quadratic, and cubic couplings and found that linear and cubic terms could modify the basic stripe or spot patterns substantially, contrary to quadratic coupling which had very little effect on the basic patterns.

More recently, a series of papers investigate a way of linearly coupling two sets of Turing equations, using the Brusselator model [11], the Oregonator model [12], or the Lengyel-Epstein model [13]. All these models preserve the stable fixed point in the absence of diffusion, and when the parameters ensure unstable resonating modes at two different wavelengths, beautiful patterns appear. Examples are the socalled "eyes," spots contained in stripes or vice versa, or patterns with spots of two different sizes (wavelengths). These models can be interpreted as systems consisting of two chemically active layers, separated by an intermediate layer that is not chemically active, but allows diffusion of chemicals through it. Experimental research has been carried out in this particular field of multilayer systems to verify the model (see [14]).

\section{B. Nonlinearly coupled Turing models}

There are also situations where the intermediate layer could be chemically active, resulting in interlayer interactions that are more complicated than simple diffusion of chemicals. For example, they may involve reactions and catalyzers. Turing systems coupled nonlinearly have been studied by us recently [15]. In this work, a cubic coupling was introduced between the constituents such that the morphogen concentration and the original fixed points of the system are conserved. The resulting fourth order characteristic polynomial may facilitate two well-separated Turing 
spaces and hence give rise to two very different wavelengths competing in a complex manner.

In contrast to the case of linear coupling, our results showed that the strength of coupling has a great impact on the patterns produced. Strong coupling between the systems leads to very simple patterns-a result of only one wavelength surviving - whereas weak coupling leads to complex patterns with symmetries that are not simply a resonant superposition of patterns with different wavelengths.

\section{Aims and outline}

The skin of vertebrates is made up of layers, which may interact by chemicals diffusing between them; as such, they are ideal systems to be modeled by coupling two sets of reaction-diffusion equations. In this paper we propose a simple model that produces patterns very similar to those observed in the skin of freshwater stingrays of the Amazon basin. We use the general reaction-diffusion system of Ref. [10] to simulate the chemical reactions in two skin layers. The kinetics are assumed to be the same in both layers and therefore events in both layers are modeled using the same set of reaction-diffusion equations, but with different parameters in each layer. To allow the diffusion of chemicals between layers one couples the two systems linearly. An important assumption is that the dynamics in one of the layers may become stationary after some time and serve as a source of morphogens for the second layer, modifying the pattern produced by the latter.

The paper is organized as follows. In Sec. II we present the biological basis of the possible mechanisms that could produce coloration of the skin and the patterns of the fish we are considering. In Sec. III we discuss the basic properties of our model system and through linear analysis we explore the various bifurcations that give rise to different patterns. In Sec. IV we exhibit the resulting patterns from extensive numerical simulations, and in Sec. V we discuss these results and draw some conclusions.

\section{BIOLOGICAL BACKGROUND}

Vertebrate skin consists of two layers: an overlying dermis separated from an epidermis by a basal lamina, see Fig. 1 and Ref. [16]. The dermis is made up of several sheets of cells, the outer sheets being formed via mitosis taking place in the inner sheets. The mesenchymal cells of the underlying dermis are able to migrate in the extracellular matrix to form structures such as the hair follicles and feather buds. The dividing basal lamina tightly couples the two skin layers whilst allowing signals to travel between them. The formation of skin appendages and patterns requires the interaction of epithelium and mesenchyme [17]. It has been demonstrated that the mesenchyme determines the number, size, location, and structural identity of the appendage, while the epithelium determines the orientation [18].

A mechanochemical framework has been suggested for modeling the formation of skin appendages and patterns [19-22]. In general these models use a system of partial differential equations to describe the density of mesenchymal

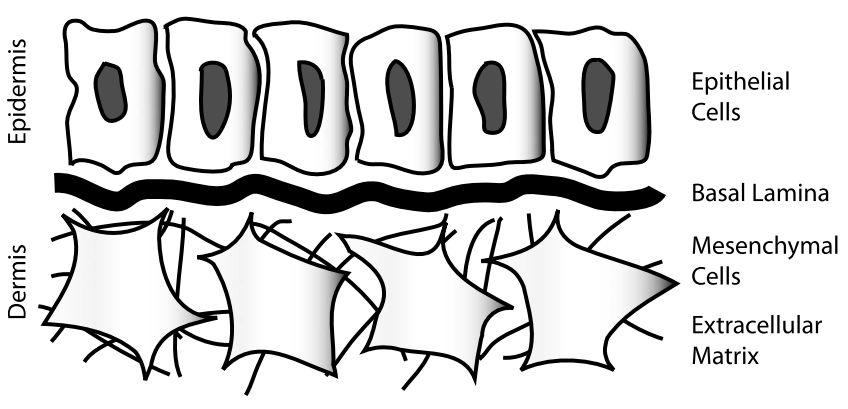

FIG. 1. Illustration of the different skin layers. The outer layer (epidermis) consists of columnar epithelial cells arranged in sheets, whilst the inner layer (dermis) consists of mesenchymal cells that are more loosely arranged and able to migrate on the extracellular matrix.

cells and extracellular matrix, with matrix deformations modeled using a small-strain assumption. Events in the overlying epidermis are modeled using a system of reactiondiffusion equations to describe the distribution of various signaling factors present. The two layers are coupled via interaction terms present in the systems. Such coupled systems are capable of producing more complex patterns than the simple systems generally used to describe patterning, but they can be difficult to study, analytically or numerically. Coupled systems of reaction-diffusion equations, however, may also lead to the generation of complex patterns and are more amenable to analytical and computational examination $[10,15]$. Our assumption in this case will be, similar to any reaction-diffusion model, that the chemical pre-pattern induces a sorting of the different cell types and therefore leads to the observed patterns.

\section{Stingray patterning}

One specific application we consider will be to pigmentation patterning in stingrays. Freshwater stingrays of the family Potamotrygonidae, the only living family of Chondrichthyes (sharks, rays, and chimaeras) restricted to freshwater, occur in many South American river systems that flow into the Atlantic Ocean or Caribbean Sea [23]. Most potamotrygonid species have highly intricate dorsal color patterns, which may include variously shaped spots, ocelli (spots surrounded by rings), rosettes, reticular, and vermiform markings, or a combination of these, usually distributed over a grey, brown, reddish-brown, or blackish background color.

Of particular interest to us will be the complex patterns found on the skin of the stingray Potamotrygon motoro, shown in Fig. 2, for three reasons: (i) In potamotrygonid stingrays, as in chondrichthyans in general, major coloration characteristics are mostly established by birth or shortly thereafter. Furthermore, coloration does not vary drastically in response to local, external stimuli, as is possible in other groups of fishes. (ii) The pattern does not look like a simple Turing pattern. (iii) The pattern differs between species and may also depend on the gender and age of the fish. We aim to show that this polymorphism could be explained by the same model. 


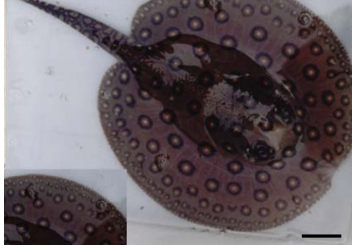

(a)

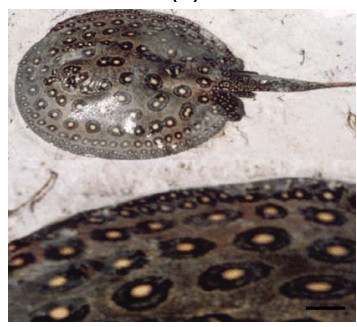

(c)

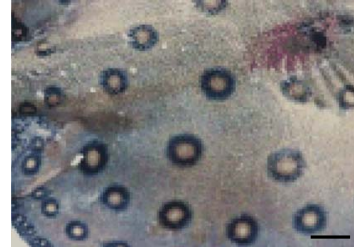

(b)

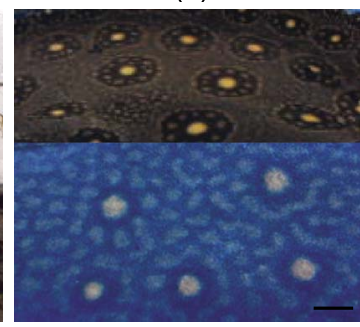

(d)
FIG. 2. (Color online) Potamotrygon motoro: (a) neonate, (b) adult female, (c) juvenile male. Potamotrygon sp.: (d) adult male. Scale bars: (a) $1.5 \mathrm{~cm}$; (b) $1.0 \mathrm{~cm}$; (c) $0.5 \mathrm{~cm}$; (d) $0.5 \mathrm{~cm}$.

\section{ANALYSIS OF THE DYNAMICAL MODEL}

In Ref. [10] we proposed a reaction-diffusion model with two chemicals, $u$ and $v$, obtained by assuming a fixed point at $(u, v)=(0,0)$ and Taylor expanding the nonlinear kinetics of the model around this point, up to cubic terms. We shall call it the BVAM model from now on. It can be written in nondimensional form as

$$
\begin{aligned}
& \frac{\partial u}{\partial t}=D \nabla^{2} u+\eta\left(u+a v-C u v-u v^{2}\right), \\
& \frac{\partial v}{\partial t}=\nabla^{2} v+\eta\left(b v+h u+C u v+u v^{2}\right),
\end{aligned}
$$

where $D=D_{u} / D_{v}$ is the ratio of diffusion constants, $\eta$ gives the spatiotemporal scale, and $C$ measures the relative strength of the quadratic and cubic terms.

The model has three fixed points located at $\left(u_{0}, v_{0}\right)$ $=(0,0)$ and $\left(u_{0}, v_{0}\right)_{ \pm}=v_{ \pm}(-g, 1)$, where

$$
v_{ \pm}=\frac{-C \pm \sqrt{C^{2}-4(h-b / g)}}{2},
$$

and

$$
g=(a+b) /(1+h) .
$$

In general, the Jacobian matrix takes the form

$$
\boldsymbol{J}=\left(\begin{array}{cc}
1-\phi & a-\psi \\
h+\phi & b+\psi
\end{array}\right),
$$

where

$$
\phi=v_{0}\left(C+v_{0}\right)
$$

and

$$
\psi=C u_{0}+2 u_{0} v_{0}=-g v_{0}\left(C+2 v_{0}\right) .
$$

The diffusion matrix is

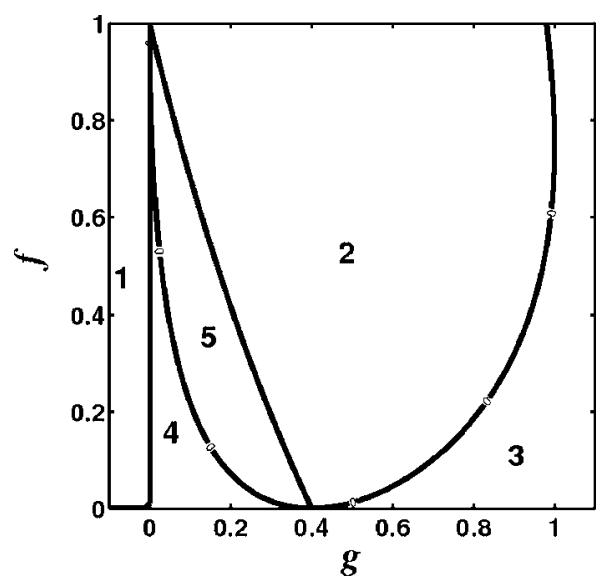

FIG. 3. Phase diagram of Eqs. (1) and (2), when $D=0, C=0$, and $h=-2.5$. In region $1, \sigma_{1}<0, \sigma_{2}>0$ and $\tau=0$ (saddle points). In region $2, \sigma_{1,2}<0$ and $\tau \neq 0$ (oscillating stable points). In region 3 , $\sigma_{1,2}<0$, and $\tau=0$ (stable points). In region $\left.4, \sigma_{1,2}>0\right)$ and $\tau=0$ (both points are unstable). In region $5, \sigma_{1,2}>0$ and $\tau \neq 0$ (oscillating unstable points). The lines were calculated by the conditions extracted from the dispersion relation Eq. (9). See text for definitions.

$$
\boldsymbol{D}=\left(\begin{array}{cc}
D / \eta & 0 \\
0 & 1 / \eta
\end{array}\right)
$$

In the linearized system a dispersion relation is found by solving the characteristic equation $|\boldsymbol{A}-\boldsymbol{\omega} \boldsymbol{I}|=0$, where $\boldsymbol{I}$ is the unit matrix and $\boldsymbol{A}=\boldsymbol{J}-\boldsymbol{D} \kappa^{2}$. One obtains

$$
\omega^{2}\left(\kappa^{2}\right)-\omega\left(\kappa^{2}\right) \operatorname{Tr}(\boldsymbol{A})+\operatorname{Det}(\boldsymbol{A})=0 .
$$

This model has only one stationary state (at $u_{0}=v_{0}=0$ ) when $h=-1$. A complete analysis of this situation, including a nonlinear analysis of the amplitude equations, has been published elsewhere [24].

The existence of the other fixed points makes this model very rich in behavior. For instance, if one fixes the value of $h$ in the absence of diffusion $(k=0)$, the complex eigenvalues $(w=\sigma+i \tau)$ can be examined in the plane $(g, f)$, where $f$ $=b / g-h$. If $\operatorname{Det}(J)=0$ one of the roots is $\omega=0$ and the other is real (observe that $h \neq-1 \Rightarrow g=0$ ). The condition $\operatorname{Tr}(\boldsymbol{J})=0$ marks the region where $\sigma$ changes sign. The discriminant condition $\operatorname{Tr}\left(\boldsymbol{J}^{2}\right)-4 \operatorname{Det}(\boldsymbol{J})=0$ separates the regions of real and complex roots. These three conditions divide the $(g, f)$ space into the five regions shown in Fig. 3.

In region 1 there are saddle points, therefore trajectories that start in the general vicinity of the fixed points first approach them, and then diverge. As a result of the cubic reaction terms, there is a stable limit cycle that encompasses the two fixed points. The existence of this limit cycle was corroborated by numerical simulation. In region 5 the fixed points are unstable; trajectories that start in their vicinity diverge in an oscillatory manner. There is also a limit cycle in this region. In region 2 both roots have negative real part, but with an oscillatory contribution. In region 3 there is the possibility of an instability driven by diffusion, producing stationary spatial Turing patterns. A detailed analysis of the model under these conditions is published elsewhere [25]. A 


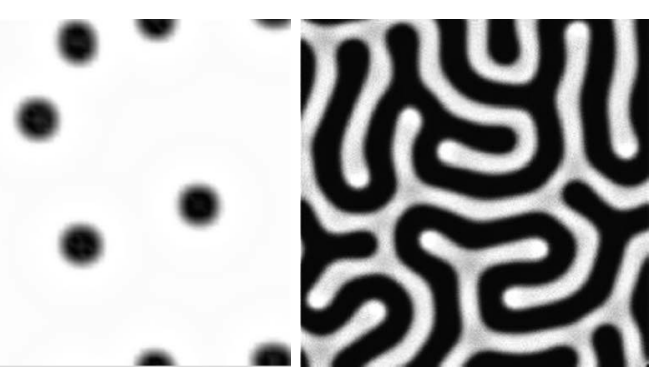

(a)

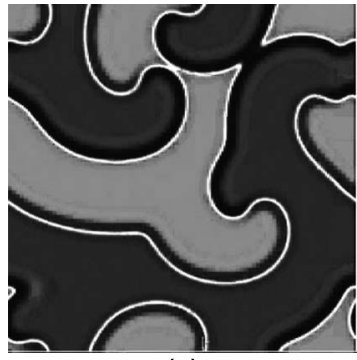

(c)

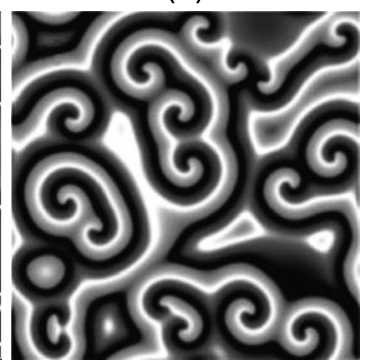

(d)

FIG. 4. Examples of patterns obtained using the BVAM model given by Eqs. (1) and (2), relaxing the condition $h=-1$ and using the parameter values detailed in the Appendix, Sec. 1. (a) and (b) are stationary, (c) shows traveling wave fronts (region 2 of Fig. 3), and (d) is an oscillating spiral pattern (region 5 of Fig. 3). In each case the domain is square with side length 254. Patterns (b), (c), and (d) were reproduced with kind permission from Springer Science +Business Media (Ref. [5], Figs. 7-9).

large variety of stationary and oscillating patterns can be obtained: in Fig. 4 we show some interesting examples.

Of particular interest are the disordered dots shown in Fig. 4(a), which are really solitary wave fronts that close in a circle of fixed radius. These structures have been studied in detail by Gomila et al. [26], examining the evolution of domain walls in nonlinear optical systems with a complex Ginzburg-Landau model. In general one can show that if a system has two different stable steady states, which are equivalent under the existing symmetries, then there are wave fronts that connect the two steady states. In a onedimensional domain they move with a velocity that depends on the shape of the profile. In two dimensions the velocity also depends on the local curvature.

These phenomena have been verified to occur in our reaction-diffusion model [25] at the border between region 2 and region 5 (see Fig. 3) in the absence of quadratic terms $C=0$, which produces two symmetrical fixed points around zero. If $C \neq 0$ the fixed points become asymmetric, and the border between regions 2 and 5 is different for each point $\left(u_{0}, v_{0}\right)_{ \pm}$. Therefore one phase will dominate over the other and circular spot patterns of one steady state solution appear embedded in the other steady state. It is these predicted stationary dots that can be seen in Fig. 4(a).

It has been shown that if certain symmetry requirements are fulfilled by the Ginzburg-Landau model the radius of the circular spot patterns can be calculated analytically [27]. The amplitude equations for the BVAM model when $h=-1$ can be written as a complex Ginzbug-Landau system [28], and it is not surprising that these robust, isolated, circular excita-

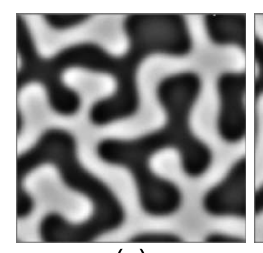

(a)

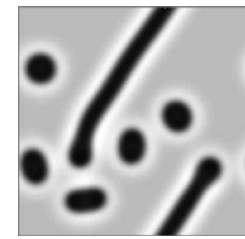

(d)

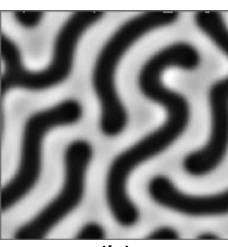

(b)

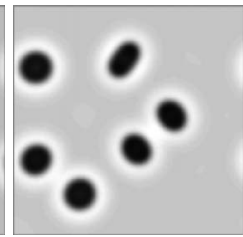

(e)

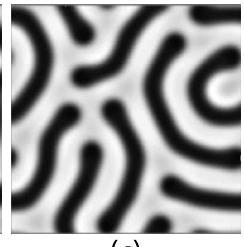

(c)

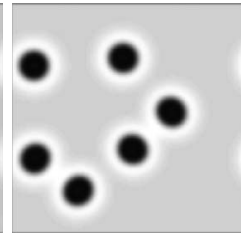

(f)
FIG. 5. Patterns obtained using the BVAM model given by Eqs. (1) and (2) and parameter values as detailed in the Appendix, Sec. 1. The values of the quadratic interaction $C$ are, respectively, 0.0 , $0.30,0.60,0.70,0.71$, and 0.72 for the images (a)-(f). In each case the domain is square with side length 80 , the same random initial conditions were used and periodic boundary conditions were imposed. The gray scale ranges from -0.4 to 0.4 .

tions appear in the BVAM model. When $h \neq-1$ and $C \neq 0$ the system loses the symmetries that allow analytical treatment of the bifurcation of Ref. [27], although it is still possible to examine this peculiar bifurcation by generalizing the method, and this work is currently in progress.

The transition from moving front waves through labyrinthine stationary patterns to disordered circular spot patterns of fixed radius when varying the value of the bifurcation parameter $C$ was studied numerically, and it is shown in Fig. 5. The critical value of $C$ lies between 0.71 and 0.72 .

All the patterns shown are stationary, except that shown in Fig. 5(a) in which the fronts move, continuously changing shape. This phenomenon arises since the velocity is proportional to the local curvature of the profiles, and as a consequence the points at which the curvature changes sign do not move, giving an impression that the fronts rotate around those points. Observe that the transition between stripes and spots is not sharp, and that one could have a combination of stripes and spots.

\section{Examples of such patterns in nature}

Patterns like these are found in many real systems, for instance the skin pattern of freshwater catfish shown in Fig. 6 varies from a purely striped pattern to a disordered pattern of dots and stripes, very similar to the ones obtained in our calculations.

\section{NUMERICAL SIMULATIONS}

We shall investigate numerically the case of a two-layered system that is coupled linearly. Evidently, the aim of this sort of research is to find new ways of simulating patterns in nature, and to gain insight into the possible mechanisms of pattern formation and selection acting on real systems. As already mentioned, complicated resonant patterns have been obtained by linearly coupling two simple Turing models, 


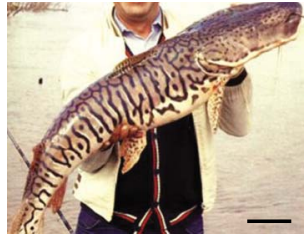

(a)

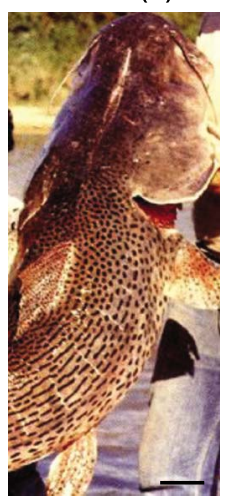

(c)

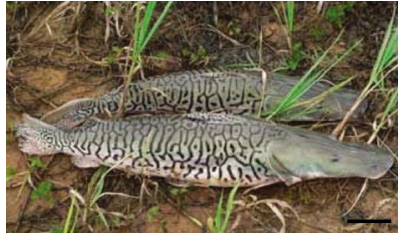

(b)

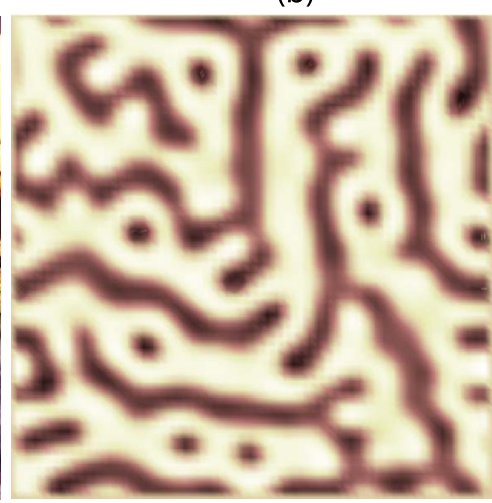

(d)
FIG. 6. (Color online) Three species of freshwater catfish in the Amazon basin, together with an example numerical simulation. (a) Pseudoplatystoma fasciatum (Surubím pintado), (b) Pseudoplatystoma tigrinum (Surubim atigrado), (c) Pseudoplatystoma coruscans (Surubim manchado). (d) A pattern obtained using the BVAM model given by Eqs. (1) and (2) and parameter values as detailed in the Appendix, Sec. 1. The domain is square with side length 80 . Scale bars: (a) $15 \mathrm{~cm}$; (b) $5 \mathrm{~cm}$; (c) $10 \mathrm{~cm}$.

each of which meet the respective Turing conditions.

Here we shall extend the numerical study of two coupled systems to the case in which one of the layers does not fulfill the Turing conditions, but produces a disordered array of circles with fixed radius. Our aim is to obtain an irregular pattern of big spots surrounded by a labyrinthine pattern as a background, as in Fig. 2. Specifically, in each layer we shall model events using the BVAM system but with the parameters in layer 1 lying in a different region of parameter space than those in layer 2.

\section{A. Two separate systems}

System 1 consists of the BVAM model of Eqs. (1) and (2) tuned with appropriate parameters to produce Turing patterns (see the Appendix for more detail). Linear analysis around the only fixed point $(0,0)$ reveals that with these parameters the complex eigenvalues $\omega_{1,2}=\sigma_{1,2}+i \tau$ have negative $\sigma$ and $\tau=0$ in the absence of diffusion. However, in the presence of diffusion, the fixed point becomes linearly unstable $(\sigma>0$ and $\tau=0$ ) to certain admissible modes around $\kappa_{c} \sim 0.65$. This is known as a diffusion-driven or Turing instability. The region of parameter space wherein the conditions of a Turing instability [10] are met is quite small for the BVAM model (see, for instance, Ref. [24]). In two dimensions it is seen that a cubic nonlinearity favors stripes, and the quadratic one produces robust spots [29]. This model produces simple Turing patterns of stripes $(C=0)$ or spots $(C \neq 0)$.

Examples of numerical calculations are given in Fig. 7 for different boundary conditions. The gray scale represents the

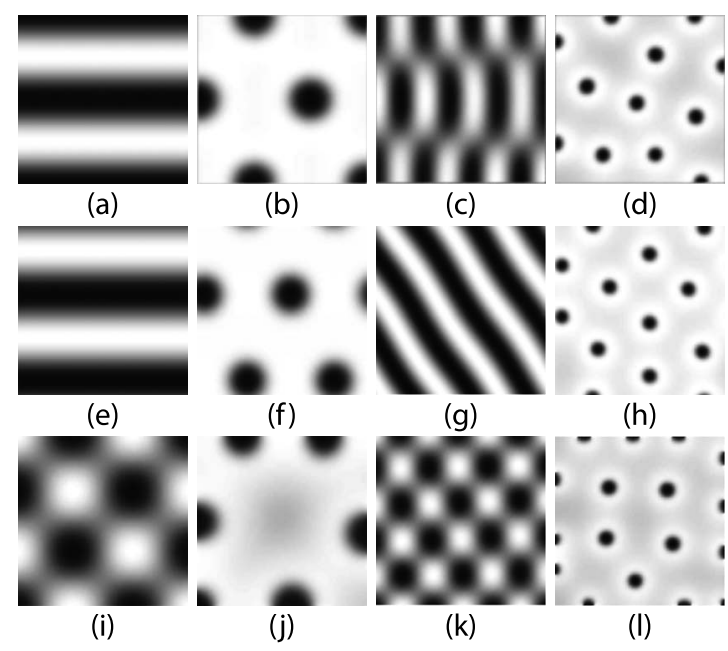

FIG. 7. Patterns in $u$ obtained from the BVAM model given by Eqs. (1) and (2) with varying parameters and boundary conditions (bc). The top row shows zero flux bc, in the second row there are periodic bc, and in the third row free bc. Columns are from left to right: first and second with a set of parameters such that $k_{c}=0.45$, third and fourth columns with $k_{c}=0.89$. In the first and third columns $C=0.0$ (favors stripes for periodic and zero flux bc), and in the second and fourth column $C=1.57$ (favors spots for periodic and zero flux bc). In all 12 patterns we used the same random initial conditions and a square domain with side length 80 . The gray scale ranges from -0.15 to 0.15 and other parameters are detailed in the Appendix, Sec. 1.

concentration of morphogen $u$. In all the numerical simulations presented here we have used a simple Euler method to integrate the dynamical equations, usually starting with a small random perturbation around zero as the initial condition. The time step has to be small enough to converge reliably [30], consequently we have used $d t \sim 10^{-2}$.

System 2 also consists of the BVAM model of Eqs. (1) and (2) but it is set up in such a way that the fixed point $(0,0)$ is unstable and the other two fixed points are stable in zero dimensions $(\kappa=0)$, but the symmetry of the two stable points is lost by setting $C=0.72$. The parameters used are detailed in the Appendix, Sec. 1, and situate system 2 at the point $(0.65,0.165)$ in the $(f, g)$ plane, near the border between region 2 and region 5 in Fig. 3. The dispersion relation for the two separate systems around the various fixed points is shown in Fig. 8.

\section{B. Two-layer coupled system}

When these two systems are coupled together, the system is now one consisting of four PDEs:

$$
\begin{aligned}
& \frac{\partial u_{i}}{\partial t}=\frac{D_{i}}{\eta_{i}} \nabla^{2} u_{i}+q_{u}\left(u_{i}-u_{j}\right)+K_{u_{i}}\left(u_{i}, v_{i}\right), \\
& \frac{\partial v_{i}}{\partial t}=\frac{1}{\eta_{i}} \nabla^{2} v_{i}+q_{v}\left(v_{i}-v_{j}\right)+K_{v_{i}}\left(u_{i}, v_{i}\right),
\end{aligned}
$$

where the reactive species $\left(u_{i}\right.$ and $\left.v_{i}\right)$ and their diffusion coefficients $\left(D_{i} / \eta_{i}\right.$ and $\left.1 / \eta_{i}\right)$ are distinguished by subscripts 


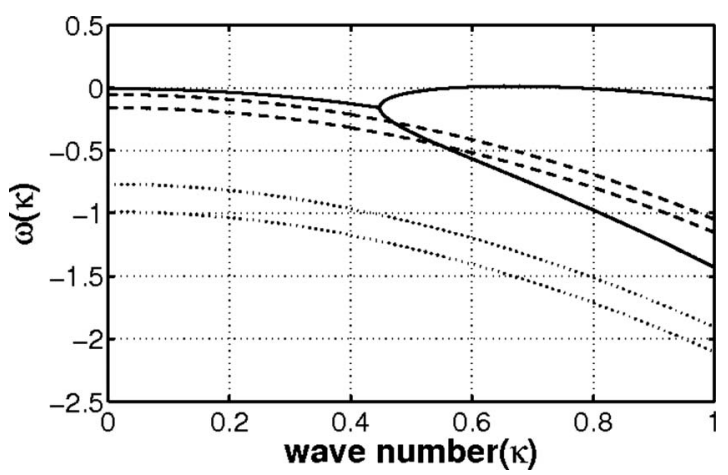

FIG. 8. The dispersion relation of system 1 for $(0,0)$ is shown using a continuous line; only the real part of the eigenvalue is shown. Around $\left(u_{0}, v_{0}\right)_{ \pm}$system 1 has complex conjugate roots, the real part around both fixed points is indicated using a dotted line. The same situation occurs for system 2 and the dispersion relation around the two fixed points is shown using dashed lines.

$i, j=1,2$ that specify the layer they are in. Both layers obey the kinetics $K_{u_{i}}$ and $K_{v_{i}}$ given in Eqs. (1) and (2), but with different parameter values. The parameters $q_{u}$ and $q_{v}$ are the coupling strengths and represent diffusion between the two layers.

Linear analysis can be carried out in the usual way. There is only one fixed point at the origin for the coupled system, namely $u_{i}=v_{i}=0, i, j=1,2$. If the coupling parameters $q_{u}$ and $q_{v}$ are small, the dispersion relation for the coupled system shows that a Turing instability is still possible. However, when the coupling increases the modes near $\kappa=0$ that are linearly stable when the systems are uncoupled, can become unstable and oscillate, and Turing patterns are not possible.

In Fig. 9 we show a series of snapshots of numerical calculations (taken at regular time intervals), of a coupled system with $q_{u}=q_{v}=0.0078$, which is the critical value for the Turing instability. We only show the dominant pattern of $u_{2}$ because $v_{2}$ is in antiphase, and because system 1 produces a fairly normal Turing pattern, as can be seen in Fig. 9(i), where the converged pattern of $u_{1}$ is shown. The parameter values used are detailed in the Appendix, Sec. 2.

Observe that the wave fronts initially moving through the domain (periodic boundary conditions were used) become immobilized when the domains of high concentration (dark regions) are approximately circular. Also notice that some of the spots split during the process. The final pattern is a random disposition of large dots, with an average distance between them much larger than the mean radius of the spots. The Turing pattern of $u_{1}$ is realized everywhere, but in the regions where there are spots the contrast is smaller, meaning that the system is closer to the bifurcation and patterns there form more slowly and with smaller amplitude. It is also noticeable that the size of the domains of high concentration of $u_{2}$ [around the value of $v_{+}$in Eq. (3)] is fairly constant throughout the domain.

The large spots are very peculiar, they are more similar to the so-called "eyes," since the shape of the wave front has a sharp asymmetric crest on the edges [25]. In Fig. 10 we show the profile of one spot taken from a cross section of the solution for $u_{2}$ shown in Fig. 9.

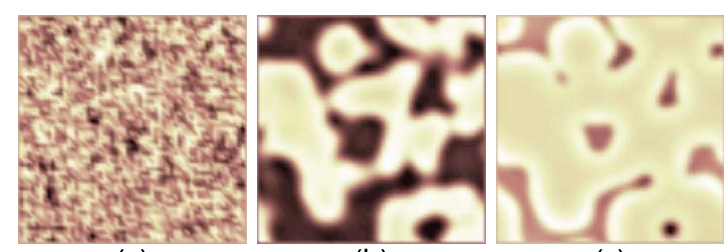

(a)

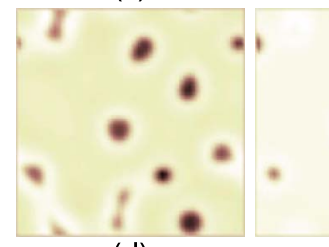

(d)

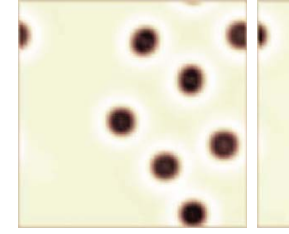

(g) (b)

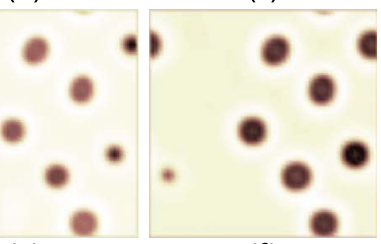

(e)

(h)

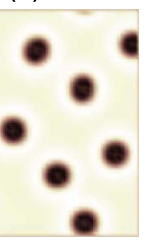

(f)

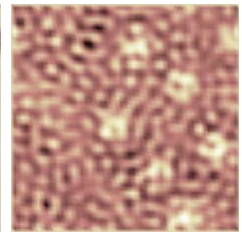

(i)
FIG. 9. (Color online) Pattern of the concentration of $u_{2}$ calculated from the two-layer coupled system given by Eqs. (10) and (11) with parameter values as given in the Appendix, sec. 2. (a) The initial random conditions. The series from (b) to (h) shows the time evolution of $u_{2}$ - snap shots are taken every 45000 time steps-and (i) is the pattern of $u_{1}$ taken at the same time as (h). The domain is square with side length 120 and the values of $u_{2}$ range from -0.4 to 0.4 (see Fig. 10).

Above the critical coupling strength no Turing pattern is formed, yet the spots of system 2 acquire a different profile, with oscillations at their borders caused by the coupling. An example of a strongly coupled system is given in Fig. 11. Observe that the pattern of $u_{1}$ is dictated by the shape of the pattern from system 2 . Only at the borders between the regions of high and low concentration (when $u_{2}$ and $v_{2}$ are near zero) does an instability appear giving rise to spatial oscillations that interfere and decay with distance.

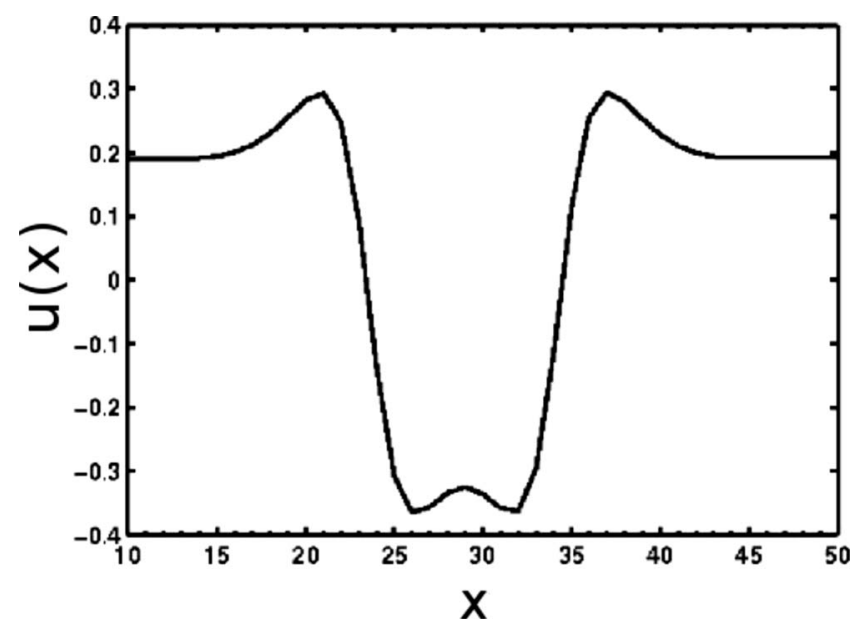

FIG. 10. Profile of $u_{2}$ along the line $y=50$ in Fig. 9(h). 


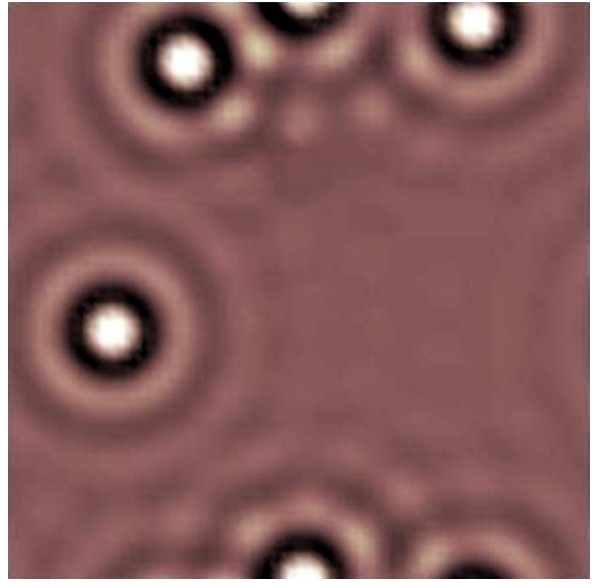

FIG. 11. (Color online) Pattern of $u_{1}$ obtained with the two-layer coupled system given by Eqs. (10) and (11) using $q_{u}=q_{v}=0.2$ and other parameter values as given in the Appendix, Sec. 2. The domain is square with side length 120 .

\section{Patterns observed in nature}

Patterns of the kind exhibited by a two-layer coupled system can be compared with the coloration of the female stingrays shown in Fig. 12. However, the coloration of the adult male fish cannot be simulated with the two layers being active. The changes seen in the coloration of the male fish as it matures suggest that at some stage in development the pigmentation pattern in one layer of the skin becomes stationary. A way of modifying the patterns is to assume that the initial pattern forms in system 2 very rapidly, and then that the dynamics become very slow on the time scale of patterning in system 1 . This could simulate the biological scenario that the cells of one of the skin layers become inactive after the animal has matured sufficiently. If this is so, the other layer could accommodate to the new situation-the inactive layer will be sensed only as a background source of morphogen on which the Turing pattern evolves.

\section{System with a source}

The hypothesis put forward for male stingray patterning can be tested by simulating the dynamics of system 1 whilst adding a constant term proportional to the final stationary pattern, $u_{2}(x, y)$, from system 2 . This will provide a complicated parameter landscape in which the pattern will evolve.

Specifically, the dynamics of system 1 after system 2 has become stationary can be followed by

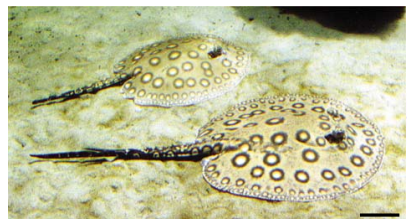

(a)

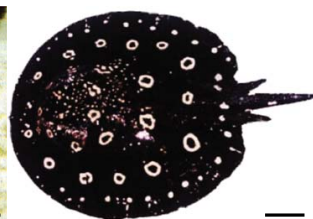

(b)
FIG. 12. (Color online) (a) Patterns seen in juvenile Potamotrygon motoro. (b) The stingray Potamotrygon leopoldi presents a coloration that looks like the color inversion of Potamotrygon motoro. Scale bars: (a) $5 \mathrm{~cm}$; (b) $10 \mathrm{~cm}$.

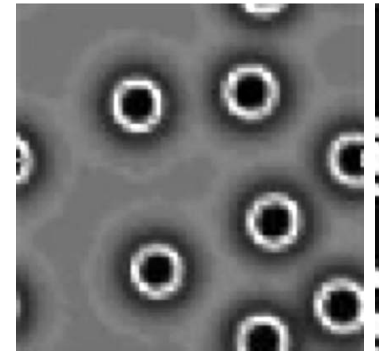

(a)

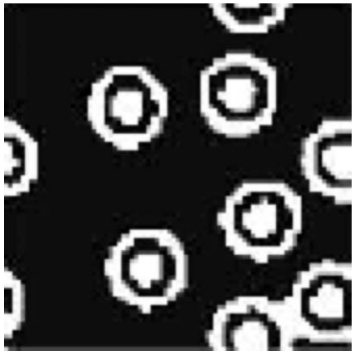

(b)
FIG. 13. (a) Pattern in $u_{2}$ used as a source and analyzed to discern the regions is which Turing instabilities can arise. (b) Black regions are places with linearly unstable modes. The domain is square with side length 120 .

$$
\begin{gathered}
\frac{\partial u_{1}}{\partial t}=\frac{D_{1}}{\eta_{1}} \nabla^{2} u_{1}+K_{u_{1}}\left(u_{1}, v_{1}\right)+q u_{2}, \\
\frac{\partial v_{1}}{\partial t}=\frac{1}{\eta_{1}} \nabla^{2} v_{1}+K_{v_{1}}\left(u_{1}, v_{1}\right),
\end{gathered}
$$

where the notation is the same as in Eqs. (10) and (11).

Linear stability analysis is now difficult: the elements of the Jacobian matrix have a space dependence because the steady states are spatially dependent due to the $u_{2}(x, y)$ term. However, as the profile $u_{2}$ is nearly constant everywhere and only varies rapidly at the edge of the circles (see profile in Fig. 10), one can apply an approximate method described elsewhere [31] when a parameter changes abruptly to predict the regions where a Turing pattern can emerge. In Fig. 13 we show the pattern used as a source, and the results of such a study.

Extensive numerical simulations were carried out guided by this analysis. We discovered that the appearance of Turing patterns is not very sensitive to the strength of the coupling, $q$, contrary to the case in Sec. IV B when both systems are active.

The patterns vary in appearance when one changes the strength of the quadratic terms $\left(C_{1}\right)$ in a way that resembles the transition from young to adult male. In Fig. 14 we show two patterns that arise from a system with a source using the same initial conditions for both. Figure 14(a) for $u_{1}$ and Fig. 14(b) for $v_{1}$ correspond to $C_{1}=0.0$. This resembles the pattern of the male stingray. Observe that although there are no quadratic nonlinearities, one obtains a dotted pattern instead of the expected Turing stripes. This is due to the interference of stripes in the complicated domain where the Turing instability exists. Figure $14(\mathrm{c})$ is obtained with $C_{1}=1.0$ and this resembles the skin of the young fish, with a mixture of spots and stripes around the large spots. Observe that the pattern for $v_{1}$ in Fig. 14(d) is in antiphase with $u_{1}$, and also with $v_{1}$ in (b). One could conclude that the different patterns the male fish exhibit are due to a degradation of some chemical reaction in the adult fish.

Other variations in the coloration of the stingrays can be simulated by coupling the system to a $u_{2}$ that is not fully converged. This could mean that the reaction in one of the skin layers stops at an earlier stage. 


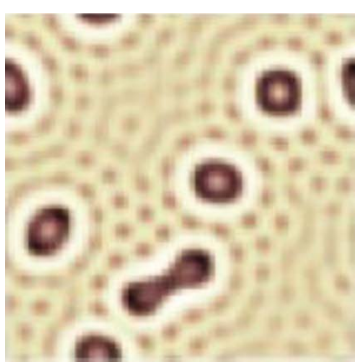

(a)

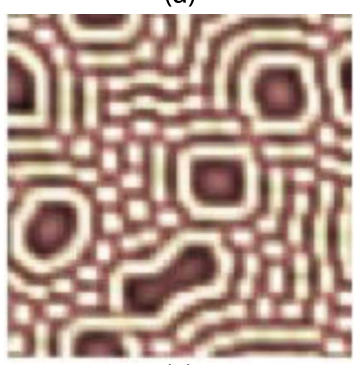

(c)

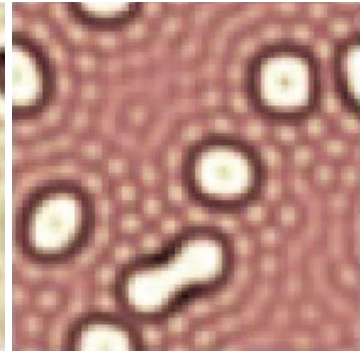

(b)

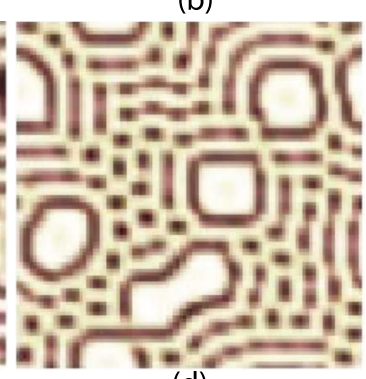

(d)
FIG. 14. (Color online) Patterns obtained from the system with a source given by Eqs. (12) and (13), calculated with $u_{2}$ as in Fig. 13 and parameters as given in the Appendix, Sec. 3. (a) $u_{1}$ using $C_{1}=0.0$, (b) $v_{1}$ using $C_{1}=0.0$, (c) $u_{1}$ using $C_{1}=1.0$, (d) $v_{1}$ using $C_{1}=1.0$. The domain is square with side length 120 and the color scale ranges from -0.4 to 0.4 . Notice that the $v_{i}$ are in antiphase with the $u_{i}$.

In Fig. 15 we show an example of a simulation carried out with a source pattern $\left(u_{2}\right)$ that has not attained the final stable values of the spot pattern, and the jump between the high and the low concentration regions of morphogen is not sufficiently large. Notice that the Turing pattern of system 1 now appears almost everywhere in the domain. In the same figure we show the time history of a single point (at the center of the domain) to reveal the way in which the dynamical system converges numerically. Notice that after a relatively small number of time steps the oscillatory convergence has attained values such that $|u(t+\delta t)-u(t)|<10^{-8}$.

Similar patterns could be obtained by diminishing the value of the coupling parameter $q$, since then one has effectively lowered the amplitude of the perturbation for system 1. In Fig. 16 we show the changes of the pattern of $u_{1}$ when one varies the coupling parameter from $q=0.0$ to $q=0.25$.
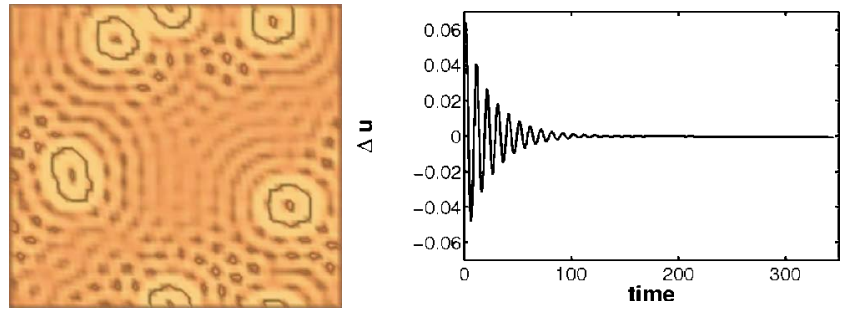

FIG. 15. (Color online) (a) Pattern obtained for the coupled system with a source when $q=0.05$ and the pattern for $u_{2}$ is not fully converged. The domain is square with side length 120 . (b) Time history of the concentration $u_{1}$ at a single point at the center of the domain, showing a rapid oscillatory convergence of the pattern.

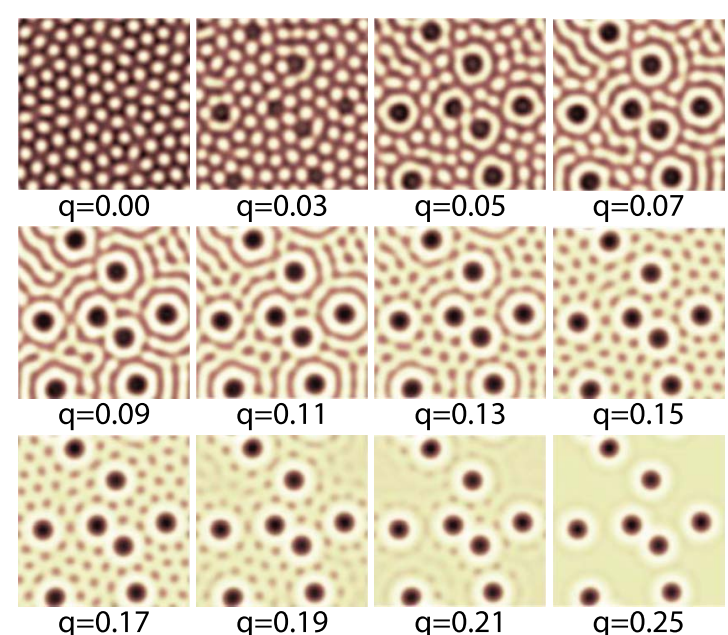

FIG. 16. (Color online) Patterns obtained from the two-layer coupled system of Eqs. (10) and (11) varying the coupling parameter $q$ from 0.00 to 0.25 . The domain is square with side length 120 and the same random initial conditions used to excite the Turing pattern when $q=0.0$ were used in every calculation. The nonlinear parameter is $C_{1}=0.5$ and the other parameters are detailed in the Appendix, Sec. 2.

\section{CONCLUSIONS}

In this paper, we have investigated pattern formation in a two-layer system in which patterning in one layer occurs on a faster time scale and serves as the driver for patterning in the layer of interest. The notion that patterns evolve from one state to another instead of emerging de novo was in fact recognized by Turing in his seminal 1952 paper [1]. Since then, a number of such studies have been proposed for patterning in the context of skin organ formation which build on this. For example, Nagorka and Mooney [32] proposed a reaction-diffusion system for hair patterning in which high concentrations of morphogen served to set up morphogen sources for subsequent patterning in the same system. This model was verified experimentally recently by Sick et al. [2]. Cruywagen et al. [33,34] proposed a coupled mechanochemical model for propagating patterns, while Painter et al. [5] coupled a chemotactic model with a Turing model to investigate complex patterning on growing domains. More recently, Page et al. [31] showed how coupled reactiondiffusion systems could lead to patterning outside the Turing regime.

A detailed numerical investigation of two-layer systems with different couplings has been carried out. These systems can generate patterns not exhibited by the one-layer systems. The novelty here is that the complexity of the underlying "forcing" system leads to quite new patterning phenomena on the overlying system. The models used to represent each layer need not be different, in fact most reaction-diffusion systems exhibit a rich variety of bifurcations that can be used. In particular, the BVAM model examined here presents Turing, Hopf, and Turing-Hopf bifurcations when $h \neq-1$. We have also shown that stationary patterns can be obtained by other means. In particular, when the system becomes bistable it is possible to obtain circular wave fronts in a stationary pattern that is extremely robust. 
We have reproduced the patterns seen in freshwater stingrays by tuning the parameters of the BVAM model to generate disordered spots and using it as a source coupled to a Turing system. This suggests that only a simple mechanism is needed to produce the changes in coloration that may be gender and age dependent, since very straight-forward variations in the concentration and diffusion of morphogens can produce the observed changes. Coloration in freshwater potamotrygonid stingrays, as in fishes and lower vertebrates in general, is provided by the uppermost strata of the dermis through pigment-containing cells known as chromatophores $[35,36]$. In our present study we have not included cells so we must think of the chemical patterns we have shown above to be pre-patterns to which such cells would respond (as in, for example, Painter et al. [5]).

Conspicuous differences in color and pattern of the skin are usually a guide for taxonomy, as in the case of freshwater catfishes in the Amazon basin. However, we have shown that diversity in coloration may be modeled by changing a single parameter in the same model, and that the patterns distinguishing various species can be obtained. Therefore a more complete understanding of color pattern formation and variation, both among and within species, as well as in relation to its ontogenetic development, is essential to properly interpret its systematic significance and usefulness in species identification. Whether species with similar color patterns are phylogenetically closely related requires the elucidation of their evolutionary history, for which molecular and morphological approaches are currently being implemented.

\section{ACKNOWLEDGMENTS}

R.A.B. is indebted to DGAPA (UNAM) and CONACyT for sabbatical grants, to CONACyT for Grant No. 79641, to the Centre for Mathematical Biology, University of Oxford and LCE, Helsinki University of Technology for their hospitality. R.E.B. and B.V. would like to thank Microsoft Research. R.E.B. would also like to thank Research Councils UK and St Hugh's College, Oxford. K.T. is grateful to ML de Araujo for support and images, and acknowledges financial support from Capes/Cnpq. M.R.C. acknowledges financial support from Fapesp Grant No. (02/06459-0). P.K.M. was partly supported by the Royal Society-Wolfson.

\section{APPENDIX: DETAILS OF THE NUMERICAL SIMULATIONS}

\section{The simple BVAM model}

The simple BVAM model can be written in nondimensional form as follows:

$$
\begin{gathered}
\frac{\partial u}{\partial t}=D \nabla^{2} u+\eta\left(u+a v-C u v-u v^{2}\right), \\
\frac{\partial v}{\partial t}=\nabla^{2} v+\eta\left(b v+h u+C u v+u v^{2}\right),
\end{gathered}
$$

\begin{tabular}{|c|c|c|c|c|c|c|}
\hline Parameter & Fig. 4(a) & Fig. 4(b) & Fig. 4(c) & Fig. 4(d) & Fig. 5 & Fig. 6 \\
\hline$D$ & 0.5160 & 0.8000 & 1.0000 & 1.0000 & 0.5160 & 1.0000 \\
\hline$\eta$ & 0.1500 & 0.5000 & 0.5000 & 0.0833 & 0.3500 & 0.3500 \\
\hline$f$ & 0.6500 & 0.7500 & 0.6543 & 0.5043 & 0.6500 & 0.6500 \\
\hline$g$ & 0.1650 & 0.1650 & 0.1650 & 0.1650 & 0.1650 & 0.1650 \\
\hline$h$ & -2.5000 & -2.5000 & -2.5000 & -2.5000 & -2.5000 & -2.5000 \\
\hline$C$ & 0.0000 & 0.0000 & 0.0000 & 0.0000 & $0.000 \rightarrow 0.7200$ & 0.8200 \\
\hline $\mathrm{BC}$ & periodic & periodic & periodic & periodic & periodic & periodic \\
\hline Grid & $256 \times 256$ & $256 \times 256$ & $256 \times 256$ & $256 \times 256$ & $82 \times 82$ & $82 \times 82$ \\
\hline Parameter & \multicolumn{2}{|c|}{ Figs. 7(a)-7(d) } & Figs. 7(e)-7(h) & Figs. 7(i) $-7(1)$ & \multicolumn{2}{|c|}{ Fig. 8 system 2} \\
\hline$D$ & \multicolumn{2}{|c|}{0.5160} & 0.5160 & 0.5160 & \multicolumn{2}{|c|}{0.5160} \\
\hline$a$ & \multicolumn{2}{|c|}{1.1123} & 1.1123 & 1.1123 & \multicolumn{2}{|c|}{0.0577} \\
\hline$b$ & \multicolumn{2}{|c|}{-1.0122} & -1.0122 & -1.0122 & \multicolumn{2}{|c|}{-0.3053} \\
\hline$h$ & \multicolumn{2}{|c|}{-1.0000} & -1.0000 & -1.0000 & \multicolumn{2}{|c|}{-2.5000} \\
\hline$\eta$ & \multicolumn{2}{|c|}{0.1000} & 0.1000 & 0.1000 & \multicolumn{2}{|c|}{0.1000} \\
\hline$k_{c}$ & \multicolumn{2}{|c|}{0.4500} & 0.4500 & 0.8900 & \\
\hline$C$ & \multicolumn{2}{|c|}{0.0000} & 1.5700 & 0.0000 & \multicolumn{2}{|c|}{0.7200} \\
\hline $\mathrm{BC}$ & \multicolumn{2}{|c|}{ zero flux } & periodic & free & & \\
\hline Grid & & & $82 \times 82$ & $82 \times 82$ & \multicolumn{2}{|c|}{$124 \times 124$} \\
\hline
\end{tabular}

TABLE I. Parameter values used in Fig. 4-8. BC indicates the choice of boundary conditions. 
TABLE II. Parameter values used in Fig. 9.

\begin{tabular}{ccc}
\hline \hline Parameter & Fig. 9 system 1 & Fig. 9 system 2 \\
\hline$D$ & $D_{1}=0.5160$ & $D_{2}=0.5160$ \\
$a$ & $a_{1}=1.1123$ & \\
$b$ & $b_{1}=-0.3053$ & \\
$h$ & $h_{1}=-1.0000$ & \\
$f$ & & $f_{2}=0.6500$ \\
$g$ & & $g_{2}=0.1650$ \\
$\eta$ & $\eta_{1}=1.0000$ & $\eta_{2}=0.1500$ \\
$C$ & $C_{1}=0.0020$ & $C_{2}=0.7200$ \\
$q_{u}$ & $q_{u}=0.0078$ & $q_{u}=0.0078$ \\
$q_{v}$ & $q_{v}=0.0078$ & $q_{v}=0.0078$ \\
\hline \hline
\end{tabular}

where $D=D_{u} / D_{v}$ is the ratio of diffusion constants, $\eta$ gives the spatiotemporal scale, and $C$ measures the relative strength of the quadratic and cubic terms. For convenience in further analysis, we define the ratios

$$
g=\frac{a+b}{1+h} \text { and } f=\frac{b}{g}-h .
$$

Table I shows the parameter values used in the BVAM model for different figures. In each case $\mathrm{BC}$ indicates the choice of boundary conditions.

\section{Two-layer coupled system}

The two-layer coupled system involves two BVAM systems coupled together via terms proportional to the difference between $u$ and $v$ concentrations in each separate system, and can be written as follows:

$$
\begin{gathered}
\frac{\partial u_{i}}{\partial t}=\frac{D_{i}}{\eta_{i}} \nabla^{2} u_{i}+q_{u}\left(u_{i}-u_{j}\right)+K_{u_{i}}\left(u_{i}, v_{i}\right), \\
\frac{\partial v_{i}}{\partial t}=\frac{1}{\eta_{i}} \nabla^{2} v_{i}+q_{v}\left(v_{i}-v_{j}\right)+K_{v_{i}}\left(u_{i}, v_{i}\right),
\end{gathered}
$$

where the reactive species are distinguished by subscripts $i=1,2$ which denote the layer they are in. The kinetic terms,

TABLE III. Parameter values used in Fig. 14.

\begin{tabular}{ccc}
\hline \hline Parameter & Fig. 14 system 1 & Fig. 14 system 2 \\
\hline$D$ & $D_{1}=0.5160$ & $D_{2}=0.5160$ \\
$a$ & $a_{1}=1.1123$ & \\
$b$ & $b_{1}=-0.3053$ & \\
$h$ & $h_{1}=-1.0000$ & $f_{2}=0.6500$ \\
$f$ & & $g_{2}=0.1650$ \\
$g$ & & $\eta_{2}=0.1500$ \\
$\eta$ & $\eta_{1}=1.0000$ & $C_{2}=0.0000$ \\
$C$ & $C_{1}=0.0020$ & \\
$q$ & $q=0.0000 \rightarrow 1.0000$ & \\
\hline \hline
\end{tabular}

TABLE IV. Parameter values used in supplementary movies.

\begin{tabular}{cccc}
\hline \hline Parameter & 0417 dots & 0417 worms & 0418 dots \& worms \\
\hline$D$ & 0.5160 & 0.3440 & 0.6450 \\
$\eta$ & 0.1500 & 0.1500 & 0.1500 \\
$f$ & 0.6500 & 0.6500 & 0.6500 \\
$g$ & 0.1650 & 0.1650 & 0.1650 \\
$h$ & -2.5000 & -2.5000 & -2.5000 \\
$C$ & 0.7200 & 0.7200 & 0.5600 \\
\hline \hline
\end{tabular}

$K_{u_{i}}$ and $K_{v_{i}}$, are as in the simple BVAM model:

$$
K_{u_{i}}\left(u_{i}, v_{i}\right)=u_{i}+a v_{i}-C u_{i} v_{i}-u_{i} v_{i}^{2}
$$

and

$$
K_{v_{i}}\left(u_{i}, v_{i}\right)=b v_{i}+h u_{i}+C u_{i} v_{i}+u_{i} v_{i}^{2} .
$$

Table II shows parameter values used in Figs. 9 and 10. The parameter values used in Fig. 11 are identical except that $q_{u}=q_{v}=0.2$.

\section{System with a source}

In the system with a source, the simple BVAM model is solved in layer 2:

$$
\begin{gathered}
\frac{\partial u_{2}}{\partial t}=D \nabla^{2} u_{2}+\eta\left(u_{2}+a v_{2}-C u_{2} v_{2}-u_{2} v_{2}^{2}\right), \\
\frac{\partial v_{2}}{\partial t}=\nabla^{2} v_{2}+\eta\left(b v_{2}+h u_{2}+C u_{2} v_{2}+u_{2} v_{2}^{2}\right) .
\end{gathered}
$$

It is then used as a source for the BVAM system used in layer 1. In general, for example in Fig. 14, system 2 is allowed to reach steady state so that $u_{2}(x, y, t) \approx u_{2}(x, y)$ before coupling to system 1:

$$
\frac{\partial u_{1}}{\partial t}=\frac{D_{1}}{\eta_{1}} \nabla^{2} u_{1}+K_{u_{1}}\left(u_{1}, v_{1}\right)+q u_{2},
$$

$$
\frac{\partial v_{1}}{\partial t}=\frac{1}{\eta_{1}} \nabla^{2} v_{1}+K_{v_{1}}\left(u_{1}, v_{1}\right)
$$

The parameter values used in Fig. 14 are detailed in Table III. The same parameters are used in Fig. 15 except that $C_{1}=0.5$ and $q$ varies between 0.00 and 0.25 .

\section{Supplementary movies}

We solve the simple BVAM model with parameter values for the supplementary movies as shown in Table IV (see Ref. [37]). 
[1] A. M. Turing, Philos. Trans. R. Soc. London, Ser. B 237, 37 (1952).

[2] S. Sick, S. Reinker, J. Timmer, and T. Schlake, Science 314, 1447 (2006).

[3] S. Kondo and R. Asai, Nature (London) 376, 765 (1995).

[4] J. L. Aragón, C. Varea, R. A. Barrio, and P. K. Maini, Forma 13, 213 (1998).

[5] K. J. Painter, P. K. Maini, and H. G. Othmer, Proc. Natl. Acad. Sci. U.S.A. 96, 5549 (1999).

[6] T. Nakamura, N. Mine, E. Nakaguchi, A. Mochizuki, M. Yamamoto, K. Yashiro, C. Meno, and H. Hamada, Dev. Cell 11, 495 (2006).

[7] A. J. Koch and H. Meinhardt, Rev. Mod. Phys. 66, 1481 (1994).

[8] H. Meinhardt, P. Prusinkiewicz, and D. R. Fowler, The Algorithmic Beauty of Sea Shells (Springer-Verlag, Berlin, 2003).

[9] H. Meinhardt, Dev. Biol. 157, 321 (1993).

[10] R. A. Barrio, C. Varea, J. L. Aragón, and P. K. Maini, Bull. Math. Biol. 61, 483 (1999).

[11] L. Yang, M. Dolnik, A. M. Zhabotinsky, and I. R. Epstein, Phys. Rev. Lett. 88, 208303 (2002).

[12] L. Yang and I. R. Epstein, Phys. Rev. Lett. 90, 178303 (2003).

[13] L. Yang and I. R. Epstein, Phys. Rev. E 69, 026211 (2004).

[14] I. Berenstein, M. Dolnik, L. Yang, A. M. Zhabotinsky, and I. R. Epstein, Phys. Rev. E 70, 046219 (2004).

[15] K. Kyttä, K. Kaski, and R. A. Barrio, Physica A 385, 105 (2007).

[16] L. Wolpert, R. Beddington, T. Jessell, P. Lawrence, E. Meyerowitz, and J. Smith, Principles of Development, 3rd edition (Oxford University Press, New York, 2006).

[17] C-M. Chuong, Molecular Basis of Epithelial Appendage Morphogenesis (Landes Bioscience, Austin, Tx, 1998).

[18] R. B. Widelitz, T.-J. Jiang, A. Noveen, C.-W. J. Chen, and C.-M. Chuong, J. Invest. Dermatol. 107, 797 (1996).

[19] J. D. Murray, Mathematical Biology (Springer-Verlag, Berlin, 2003).

[20] J. D. Murray, P. K. Maini, and R. T. Tranquillo, Phys. Rep. 171, 59 (1988).

[21] J. D. Murray, G. F. Oster, and A. K. Harris, J. Math. Biol. 17,
125 (1983)

[22] G. F. Oster, J. D. Murray, and A. K. Harris, J. Embryol. Exp. Morphol. 78, 83 (1983).

[23] M. R. de Carvalho, N. R. Lovejoy, and R. S. Rosa, Checklist of the Freshwater Fishes of South and Central America, Family Potamotrygonidae (River Stingrays), Porto Alegre, 2003, pp. 22-28.

[24] T. Leppänen, Ph.D. thesis, Helsinki University of Technology, Helsinki, 2004.

[25] C. Varea, D. Hernández, and R. A. Barrio, J. Math. Biol. 54, 797 (2007)

[26] D. Gomila, P. Colet, G.-L. Oppo, and M. S. Miguel, Phys. Rev. Lett. 87, 194101 (2001).

[27] D. Gomila, Ph.D. thesis, Universitat de les Illes Balears, 2003.

[28] R. A. Barrio, Turning Systems: A General Model for Complex Patterns In Nature In Physics of Emergence and Organization, edited by I. Licata and A. J. Sakaji (World Scientific, Singapore, 2007), 267-296.

[29] B. Ermentrout, Proc. R. Soc. London, Ser. A 434, 413 (1991).

[30] J. L. Aragón, M. Torres, D. Gil, R. A. Barrio, and P. K. Maini, Phys. Rev. E 65, 051913 (2002).

[31] K. M. Page, P. K. Maini, and A. M. Monk, Physica D 202, 95 (2005)

[32] B. N. Nagorcka and J. R. Mooney, J. Theor. Biol. 98, 575 (1982).

[33] G. C. Cruywagen, P. K. Maini, and J. D. Murray, IMA J. Math. Appl. Med. Biol. 9, 227 (1992).

[34] G. C. Cruywagen, P. K. Maini, and J. D. Murray, SIAM J. Appl. Math. 61, 213 (2000).

[35] Traité de Zoologique. Tome XIII. Agnathes et Poissons, Anatomie, Éthologie, Systématique, Premier Fascicule, edited by P. P. Grassé (Masson \& Cie, Paris, 1958), Chap. 5, pp. 433-458.

[36] J. J. Nordlund, R. E. Boissy, V. J. Hearing, R. A. King, and J.-P. Ortonne, The Pigmentary System: Physiology and Pathophysiology (Oxford University Press, Oxford, 1998), Chap. 3, pp. 9-40.

[37] See EPAPS Document No. E-PLEEE8-79-044903 for supplementory movies. For more information on EPAPS, see http:// www.aip.org/pubservs/epaps.html. 